\title{
A Comparison of Ziehl-Neelsen Staining and Fluorescent Microscopy for Diagnosis of Pulmonary Tuberculosis
}

\author{
Roma Goyal $^{1,}$ Anil Kumar ${ }^{2}$ \\ 1. Demonstrator, Dept. of Microbiology, Muzaffarnagar Medical College, Muzaffarnagar. \\ 2. Demonstrator, Dept. of Microbiology, Muzaffarnagar Medical College, Muzaffarnagar.
}

\begin{abstract}
According to WHO one third of the world population have tuberculosis. The present study was undertaken to compare the efficacy of flurochrome(FI) stain with conventional Z-N stain in the diagnosis of pulmonary \& extra- pulmonary tuberculosis.

388 cases of suspected pulmonary tuberculosis were included in the study. All samples were screen for Acid Fast Bacilli (AFB) by Z-N \& Fluorescent staining methods. Positive samples detected by fluorescent stain were $57(14.69 \%)$ when compared to Zn stain 29(7.47\%).
\end{abstract}

Conclusion: Compared to Z-N stain (7.47\%) flurochrome staining was found to be more efficient (14.69\%) in AFB detection of AFB from cases of Pulmonary Tuberculosis.

Key Words: Pulmonary tuberculosis, flurochrome stain, Z-N stain.

\section{Introduction}

Pulmonary tuberculosis is mainly a disease of the respiratory system, caused by Mycobacterium tuberculosis .Tuberculosis is a predominant infectious cause of mortality today [2,5]. According to World Health Organization (WHO), tubercular infections are currently spreading at the rate of one person per second per million people. With three millions dying from it $[1,2]$. Tuberculosis continues to be a major health problem in our country and is the single largest cause of loss in healthy life year in the productive age group. There are various methods for bacteriological diagnosis of tuberculosis [21]. Currently, radiometric assay allows detection of Mycobacterium tuberculosis growth and provides antibiotic sensitivity results more rapidly usually within 10 days. However use of the technique is limited because culture medium contains radioactive carbon. Genetic probes are on the other hand quite easy to use and allow identification of culture bacteria in only a few hours by polymerase chain reactions. Mycobacterium tuberculosis strains can be detected directly in the sputum specimen within 2 or 3 hours, but in practice, this method has not become a routine laboratory technique, particularly due to lack of sufficient specificity and sensitivity. Serological tests are currently not reliable enough for the diagnosis of tuberculosis [2]. Microscopic examination and culture are still essential elements of the bacteriological diagnosis of tuberculosis in microscopic examination; the diagnosis of tuberculosis is confirmed on the basis of demonstration of tubercle bacilli in the sputum or any other pathological material [1, $2,4,6]$. Smear examination is believed to be simple, cheap, quick and practicable and effective case finding method for developing countries. As tuberculosis bacilli are very slow growing organisms, culture results are available after a period of three or six weeks.

So, Microscopic examination has the advantage of the giving a result at once. The specimen most commonly examined is sputum and mucous secretion coughed up from the lungs [5].

Microscopic examination of Ziehl-Neelson or auramine stained specimen allows detection of most strains in less than an hour.

Ziehl-Neelson is the most extensively used procedure for the demonstration of mycobacterium tuberculosis in smear [7,8]. The requisites for the staining procedures are; basic fuchsin, phenol, absolute alcohol; sulphuric acid and methylene blue. Microscopic examination under oil immersion objective reveals mycobacterium are red bacilli.

Fluorescent staining by Auramine is other methods of staining. In this a smear is made from the specimen and stained with fluorescent stain called auramine. The auramine stain enters the wall of Mycobacterium tuberculosis bacterial cell and makes them glow against dark background under UV light [4].Microscopic examination under low power objective will reveal mycobacteria as glowing yellow white, rice like bacteria in the smear,.

Therefore the present prospective study was under taken to see the efficacy of Ziehl-Neelson method versus fluorescent staining in the detection of mycobacterium in sputum sample. 


\section{Material And Methods}

The comparative study was conducted in Meerut city from 19.3.2011 to 19.9.2011. Total 388 (negative, scanty, 1+) sputum samples were collected from the Direct Observation Treatment Short course (DOTS) center, Meerut.

PATIENTS INCLUSION CRITERIA: Patients attending the DOTS Centre, having fever, night sweats, cough for more than 3 weeks with sputum, loss of appetite, loss of weight, chest pain, heamoptysis and/ or radiological evidence of tuberculosis were included.

SAMPLE COLLECTION: Early morning sputum samples were collected in clean, sterile, leak proof, wide mouth containers. The processing of the sample were carried out in a biosafety cabinet. each sample was subjected to Ziehl-Neelson(ZN) staining and fluorescent Auramine-O (AO)staining

Table1. Quantization scale for acid-fast bacillus smears according to Forbes BA et al.

\begin{tabular}{|l|l|l|}
\hline $\begin{array}{l}\text { Carbolfuchsin } \\
\text { (X1,000) }\end{array}$ & $\begin{array}{l}\text { Fluorochromes } \\
\text { (X 250) }\end{array}$ & Quality reported \\
\hline No AFB/300 fields & No AFB/30 fields & No AFB seen \\
$1-2 \mathrm{AFB} / 300$ fields & $1-2 \mathrm{AFB} / 30$ fields & Doubtful (repeat test) \\
$1-9 \mathrm{AFB} / 100$ fields & $1-9 \mathrm{AFB} / 10$ fields & Rare $(1+)$ \\
$1-9 \mathrm{AFB} / 10$ fields & $1-9 \mathrm{AFB} / 1$ fields & Few $(2+)$ \\
$1-9 \mathrm{AFB} /$ field & $10-90 \mathrm{AFB} /$ field & moderate $(3+)$ \\
$>9 \mathrm{AFB} /$ field & $>90 \mathrm{AFB} /$ field & numerous $(4+)$ \\
\hline
\end{tabular}

Table II-Result of smear examination by Ziehl-Neelson and Fluorescent staining

\begin{tabular}{|l|l|l|}
\hline & ZN- STAINING & FLOURESCENT STAINING \\
\hline NEGATIVE & $359(92.53 \%)$ & $331(85.31 \%)$ \\
\hline POSITIVE & $29(7.47 \%)$ & $\mathbf{5 7}(14.69 \%)$ \\
\hline TOTAL & \multicolumn{1}{|c|}{$\mathbf{3 8 8}(\mathbf{1 0 0 \%})$} \\
\hline
\end{tabular}

Table III- Comparison of smear examination result by $\mathrm{ZN}$ staining and fluorescent staining

\begin{tabular}{|c|l|l|l|}
\hline & ZN-POSITIVE & ZN-NEGATIVE & TOTAL \\
\hline Flourescent positive & 29 & 28 & 57 \\
\hline Fluorescent negative & 0 & 331 & 331 \\
\hline Total & 29 & 359 & 388 \\
\hline
\end{tabular}

Table IV- Correlation of ZN staining and fluorescent staining gradewise.

\begin{tabular}{|c|l|l|}
\hline \multirow{2}{*}{ GRADING } & \multicolumn{2}{|c|}{ POSITIVE BY } \\
\cline { 2 - 3 } & ZN STAINING & FLOURESCENT STAINING \\
\hline SCANTY & 4 & 10 \\
\hline $1+$ & 25 & 47 \\
\hline TOTAL & $29(7.47 \%)$ & $57(14.69 \%)$ \\
\hline
\end{tabular}

A total of 388 clinically diagnosed pulmonary tuberculosis patients were included in the study Out of 388 sputum samples, the smear positivity for AFB on the conventional ZN method was 7.47\% (29/388) while the positivity increased to $14.69 \%$ (57/388) on the modified fluorescent method.

Table 2 shows, the correlation between the conventional ZN method and the modified fluorescent method. The ZN smear positivity rate and the AO smear positivity rate in this study was $7.47 \%(29 / 388)$ and $14.69 \%(57 / 388)$ respectively.

Table 3: shows 29 samples were both fluorescent and $\mathrm{ZN}$ positive, where 28 samples were Fluorescent positive and $\mathrm{ZN}$ negative. Out of which 331 samples were both fluorescent and ZN negative. Where none of the sample was ZN positive and fluorescent negative respectively.

Table 4: shows that scores are definitely higher by fluorescent microscopy: $57(10+47)$ positive as against $29(4+25)$ positive by $\mathrm{ZN}$ staining. 
Figure 1 shows Acid Fast Bacilli (1+) with flourescent staining.

Figure 2 shows Acid Fast Bacilli (1+) with Zeihl Neelson's staining.

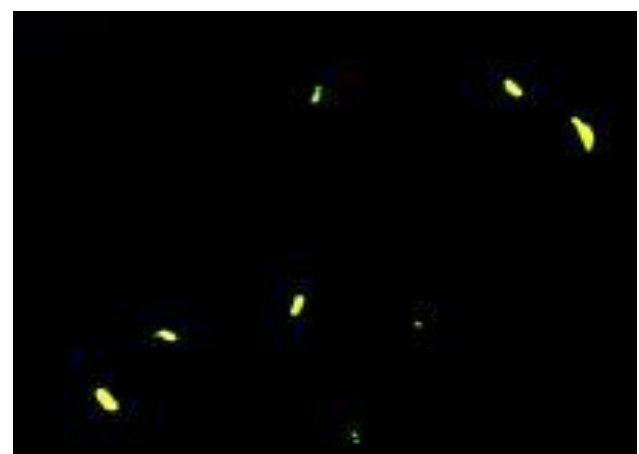

Figure 1

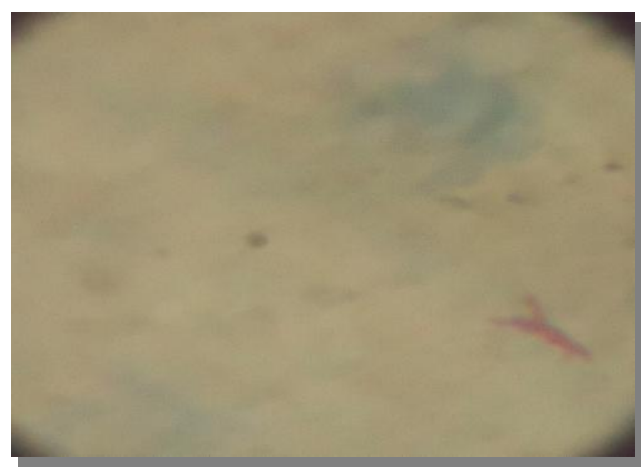

Figure 2

\section{Discussion}

India has a long history of research and demonstration projects on TB. The detection of Acid fast bacilli is often considered as the evidence of the infected stage. Thus, the laboratory plays a critical role in the diagnosis of TB [3]. In developing countries, microscopy of the specimen is by far the fastest, cheapest, and most reliable method for the detection of AFB [5,6]. However fluorescent staining has been added in Revised National Tuberculosis Control Program (RNCP) because of more sensitive and rapid results and can be used in field areas.

In the present study, the results showed that from sputum specimen of 388, 29 patients had sputum smear positive by $\mathrm{ZN}$ staining and by fluorescent staining 57 patients gave positive result [9].

The results of present study indicate that Auramine staining of sputum smears in is a more sensitive method of sputum microscopy for demonstration of AFB in sputum specimen, compared to Ziehl-Neelson staining.

The use of Fluorescent Microscopy greatly improves the diagnostic value of sputum smear especially in patients with low density of bacilli that are likely to be missed on Zeihl Neelson stained smears. The method is economical in both time and expense and recommended for laboratories handling large number of sputum specimens [7].

Fluorescent staining is superior to that of ZN staining in the presence of a low bacterial load as seen in smears with diagnostic cytomorphologial featured tuberculosis, in problem areas like AIE (acute inflammatory exudates alone or with occasional granulomma, AFB positivity by $\mathrm{ZN}$ staining is nearly as good as the fluorescent method because bacterial load is high) $[12,13]$.

Using fluorescent microscopy, the tubercle bacilli when examined under ultra violet illumination, the bacilli appeared as a bright rod against a dark back ground. Since there was a contrast, the bacilli were readily seen and therefore in very less time large area could be examined.

Images were then captured with the digital camera and enhance through imaging processing techniques were captured.

While in $\mathrm{ZN}$ staining acid fast bacilli appeared bright red rods in blue background. In this also image

The potential benefits of automated screening for tubercle bacilli are: rapid, acute, inexpensive diagnosis; the ability to screen large number of people; increased resources to monitor patients; and reduction in health risk to staff.

Thus the study reveals that sputum stained by the florescent method is useful and reliable for pulmonary tuberculosis.

Since the fluorescent microscopy is costly some laboratories cannot afford to buy florescent microscopy, so in these laboratories Ziehl-Neelson staining is most employed [14,15]. 


\section{Conclusion}

Sputum examination for the tubercle bacilli is usually conducted for patients clinically and/ radiologically suspected of pulmonary tuberculosis. However, the standard method of sputum examination, that is, $\mathrm{ZN}$ staining is not sensitive enough and a large number of the suspected cases miss diagnosis. Moreover many cases remain unsuspected and don't seek treatment.

Fluorescent stain is a more efficient over ZN stain in detecting Tubercle bacilli in sputum. Since screening is done under low power of magnification (40X), fluorescence has been found to be less time consuming compared to ZN method (100X) in the diagnosis of tuberculosis. Hence, it has been advocated to be methods of choice where the large numbers of sputum smears are to be examined. The fluorescing bacilli are easily identifiable and cause less eye-strain.

\section{Refrences}

[1]. Ayahs Gupta, S. K. sharma and J.N Pande(1993) Diagnostic methods for tuberculosis, the India Journal of Chest Diseases and Allied Services.

[2]. Ba F and Rieder HL (1999) A comprision of flurescence microscopy with the Zeihl-Neelson technique in the examination of sputum for acid-fast bacilli, Int J Tercle Lung Disease.; 3 (12): 1101-5

[3]. Clancey JK, Allen BW, Rogers DT, Smith LS, Aber V and Mitchison DA (1976) Comparison of machine and manual staining microscopy, J Clin Pathol.; 29 (10); 931-3

[4]. Claxton PD, Eamens GT and Mylrea PJ (1979), Laboratory diagnosis of bovine tuberculosis, Auet 1 Vet J.; 55 (11):514-20

[5]. Desgmukh SR, Mantri SB, Kendre PB and Nagoba BS (1996) A comparison of sputum examination for acid fast bacilli by modified Schaeffer and fulton stain, Zeihl-Neelson stain and cold stain, Indian J Med Res.; 103; 294-5.

[6]. 6. Dandapat MC, Mishra BM, Dash SP, Kar PK. Peripheral lymph node tuberculosis. A review of 80 cases. Br J Surg 1990;77:911-2.

[7]. Lau SK, Kwan S, Lee J, Wei WI. Source of tubercle bacilli in cervical lymph nodes: A prospective study. J Laryngol Otol 1991;105:558-61.

[8]. Pamra SP, Mathur GP. A co-operative study of tuberculous cervical lymphadenitis. Indian J Med Res 1974;62:1638-46.

[9]. Paria KK, Gosh RK, De PK, Sengupta J, Mukherjee AC, Pradhan MC. Study on clinically diagnosed tuberculous cervical lymphadenitis not responding to standard antituberculous chemotherapy. Indian J Tuberc 1985;32:133-44.

[10]. Daniel TM. Rapid diagnosis of tuberculosis: Laboratory techniques applicable in developing countries. Rev Infect Dis 1989;2:471 8 .

[11]. Balows A, Hausler WJ, Herrmann KL, Shadomy HJ. In: Manual of clinical Microbiology. 5th ed. American Society for Microbiology. Washington: D.C: 1991. p. 308-11.

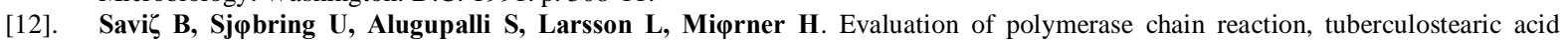
analysis, and direct microscopy for the detection of Mycobacterium tuberculosis in sputum. J Infect Dis 1992;166:1177-80.

[13]. Central tuberculosis division. In: Tuberculosis - A guide for practising physicians. Revised National Tuberculosis Control Programme, Directorate General of Health Services: New Delhi: 2004. p. 1-5.

[14]. Tarhan G, Ordulu L, Gümü?lü F, Ceyhan I, Cesur S. Comparison of auramine-rhodamine and Erlich-Ziehl-Neelsen staining methods for the diagnosis of tuberculosis. Mikrobiyol Bul 2003;37:131-6.

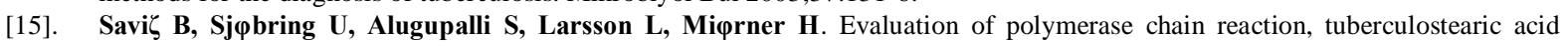
analysis, and direct microscopy for the detection of Mycobacterium tuberculosis in sputum. J Infect Dis 1992;166:1177-80 